Copyright (C) 2021 by Cherkas Global University

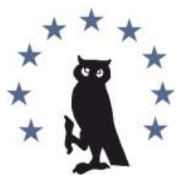

Published in the USA

European Journal of Medicine

Has been issued since 2013 .

E-ISSN: 2310-3434

2021. 9(2): $28-36$

DOI: 10.13187/ejm.2021.2.28

https://ejm.cherkasgu.press

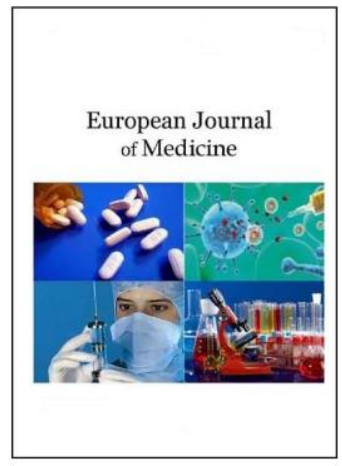

\title{
Articles
}

\section{Probiotic Effects on Covid-19}

\author{
Rana H. Raheema a ${ }^{\text {, }}$, Marwa A. Al-Asady a \\ ${ }^{a}$ College of Medicine, Wasit University, Iraq
}

\section{Abstract}

This study focuses on the impacts of probiotics on covid-19 infection. Probiotics have the ability for enhancing gastrointestinal tract health, modulating the immune system and reducing the occurrence of allergy in susceptible people.

In addition, probiotics are capable of supporting the host's immune system to fight viral infections. There is a relationship between COVID-19 disease and intestinal dysbiosis, also there are close links between gastrointestinal tract and respiratory tract infection.

Probiotics can restore the composition of the gut microbiota "eubiosis", also regulating the immune response in respiratory tract infection and acts as an anti-inflammatory that can reduce the inflammation/cytokine storm and other symptoms (vomiting, diarrhea) in COVID-19 infection.

Also, strains that are related to the lactic acid bacteria (probiotic) might change the human intestine/gut microbiota through opportunistic bacteria growth suppression. Thus, stimulation and administration of the activity and growth of probiotic strains in intestine/gut might be specified as possible method for controlling food borne enteric pathogens.

There were numerous health advantages to the probiotics elsewhere in the gut; the probiotics have exposed for improving the immunity, reducing severity regarding specific allergic conditions and deliberate a few anti carcinogenic characteristics.

Keywords: probiotics, COVID-19, Immunity, Lactobacillus spp.

\section{Introduction}

Probiotics are defined as live microorganisms which, in the case when being administered in passable amounts, provide many health benefits to the host (Hill et al., 2014).

Lactobacillus species, Saccharomyces and Bifidobacterium were the most prevalent microorganisms as probiotic (Guimaraes et al., 2020; Zendeboodi et al., 2020).

They were commercially available as added to certain dairy products and food, also supplements (Kiousi et al., 2019; de Almada et al., 2015; Roobab et al. 2020). There were prebiotics, which have been substrates consumed selectively via the host micro-organisms and conferring health benefits (NeriNuma et al., 2020). Also, strains that are related to the lactic acid bacteria (probiotic) might change the human intestine/gut microbiota through opportunistic bacteria growth suppression. Thus, stimulation and administration of the activity and growth of probiotic strains in intestine/gut might be specified as possible method for controlling food borne enteric pathogens.

\footnotetext{
${ }^{*}$ Corresponding author

E-mail addresses: rraheema@uowasit.edu.iq (R.H. Raheema)
} 
There were numerous health advantages to the probiotics elsewhere in the gut; the probiotics have exposed for improving the immunity, reducing severity regarding specific allergic conditions and deliberate a few anti carcinogenic characteristics. There were limited data provided regarding the effects of COVID19 upon the intestinal microbiota (Khaneghaha et al., 2020). SARS-CoV2 might proliferate in gastrointestinal and respiratory tracts (Lamers et al., 2020). It has been indicated that COVID19 is affecting the functioning and composition of digestive tract; thus, changing the gut microbiota for long periods (Mohanty, Dhar, 2020).

A few researches showed that the probiotics might have possible anti-viral and antiinflammatory effects. They are acting via the suppressing production of cytokines whether locally, for instance, on levels of the intestinal mucosa, or on extra intestinal organs of the body. Many medical trials specified that the probiotics' administration to enhance the effects in conditions that are associated with the immune system as well as viral infection (Kiousi et al., 2019).

\section{SARS-COV-2 (COVID-19 infection)}

Coronaviruses are specified as members of the enveloped positive sense viruses of the RNA, belonging to Nidovirales order that is related to Coronaviridae family. Also, the distinctive properties of glycoprotein spikes on the surface of the virus are promoting its easy adhesion and identification with the cells of the host. Corona viral RNA has been large up to $32 \mathrm{~kb}$ (Hulswit et al., 2016).

Also, the coronavirus that is associated with the animal and human hosts has been classified to 4 genera, delta, gamma, beta and alpha viruses. In addition, the alpha coronavirus involves human isolates Human Coronavirus 229E (HCoV-299E) and HCoVNL63, while beta coronavirus includes $\mathrm{HCoV}-\mathrm{OC} 43$, HCoVHKUL and the recently-included SARS-CoV, MERS-CoV, and SARSCoV-2 (COVID-19) (Zaki et al., 2012; Drosten et al., 2003; Zhu et al., 2020a). COVID-19, the latest CoV outbreaks that was started in Wuhan, China in 2019, is the results of SARS-CoV-2 (Gorbalenya et al., 2020; Zhou et al., 2020a). The virus was categorized in family Coronaviridae, order Nidovirales, Beta-coronavirus genus and subfamily Coronavirinae. On December 2020, over 50 million SARS-Cov-2 infections were identified all over the world.

To initiate the infection of SARS-CoV-2 adhering to the ACE2, receptor on epithelial cells that are related to hosts via their surface glycoprotein spikes, S1 and S2 (Hoffmann et al., 2020).

In addition, ACE2 has been expressed mostly in lung type-II pneumocytes, colon colonocytes, cholangiocytes, ileum endothelial cells, esophagus keratinocytes, stomach epithelial cells, kidney proximal tubules, and rectum endothelial cells (Qi et al., 2020). N-terminal S-1 portion regarding viral S protein is of high importance in the targeting of host cell receptor ACE-2. Receptor binding was facilitated through C-terminal receptor binding domain on S 1 portion (Wu et al., 2009; Li et al., 2005; Reguera et al., 2012; Li, 2012). Following the binding of the receptor through $\mathrm{S} 1$ portion, $\mathrm{S} 2$ portion facilitate fusion between viral as well as host cell membrane. Also, $\mathrm{S} 2$ portion has many fusion peptides and 2 conserved repeats of the heptad that were vital in direction finding in addition to the fusion of virus via cell membrane ( $\mathrm{Lu}$ et al., 2015). After SARS-CoV2 genome release to the host cell's cytoplasm, viral RNA will be replicated and following (2-14) days of the incubation, the symptoms of infection will emerge, yet some targets are without symptoms, and scanning might be confirming infection alone (Fanos et al., 2020).

Throughout the start of sickness, the major symptoms shown via the majority of patients were cough and fever. Other symptoms such as fatigue or muscle pain (myalgia), conjunctivitis, shortness of breath (dyspnea), headache, diarrhea, chest pains, runny nose (rhinorrhea), nausea, vomiting, gastrointestinal bleeding, loss of appetite, abdominal pains, coughing of blood (hemoptysis) and autoimmune hemolytic anemia (D'Amico et al., 2020; Huang et al., 2020; Chen et al., 2020b; Wang et al., 2020b; Dockery et al., 2020; Kopel et al., 2020; Lazarian et al., 2020).

Also, patients have stated dysgeusia (distortion of taste sense) and anosmia (loss of smell) (Azez et al., 2020; Hopkins et al., 2020; Mermelstein, 2020; Nunan, 2020). With regard to asymptomatic patients of SARS-CoV2, anosmia, dysgeusia, or hyposmia were the symptoms which have been suggestive of the screening (Lao et al., 2020).

SARS-CoV2 is majorly transmitted through respiratory droplets from the infected individuals, through the eyes and by contact with the surfaces that have been contaminated by virus (Hamid et al., 2020).

\section{Immune response in COVID-19}

Like all infections, the innate/adaptive immune system should be mounting effective defenses against a viral invasion. COVID-19 has been reported circulating T-helper cells (CD $4+$ 
cells), B-cells, Cytotoxic T-cells (CD 8+ cells), lymphocytes, natural killers' cells (NKs), eosinophils, monocytes and basophils are declined (Kuri-Cervantes et al., 2020; Wu et al., 2020c; Xu et al., 2020b; Huang et al., 2020).

The immunity against SARS-CoV-2 antigen gets functional loss. Dysregulation that is related to macrophage and monocyte function is promoting a marked decrease in CD16+ monocytes as well as an insignificant increases in CD14+ monocytes (Wilk et al., 2020).

In addition, the up regulation of CD14+ monocytes could be because of the secretion of IL6 through inflammatory monocytes. (Zhou et al., 2020). The accumulations of the macrophages at infection site are stimulating the fibroblasts to result in pulmonary fibrosis (PFs). Also, peripheral NKs are losing their functional impacts in the severe SARS-CoV2 infections and have been decreased, while there was an increase in lymphopenia and apoptosis (Xiong et al., 2020).

DCs are capturing the viral antigen as well as presenting it to T-cells which exist in lymphoid tissues and mucus epithelium. Also, they are secreting cytokines for regulating immune responses (I.R) as well as maintaining the homeostatic equilibrium which is disturbed because of virus infection. Along with phagocytosis, the DCs are inducing adaptive immunity towards the viral antigens.

Plasmacytoid DCs (pDC) and myeloid DCs (mDCs) have activated anti-viral responses through creating a considerable amounts of type-I interferon (INF) and making immune surveillances in air route as well as distal lung via intrinsic, innate receptors, with the use of RIG-1, MDA5, RNA sensing TLRs7 \& 8 and NLRP3 inflammasome (Gupta et al., 2020). Throughout presentation of antigen to Tcells, APC has been extremely specific. In the case where it is interacting with CD 4+ T-cells, it will be differentiated to Th cells of various types Th2, Th 1, Th17 or other CD4+ T-cells. Its secretions, like chemokines and cytokines are influencing such T-cell process. With regard to the differentiation of naive CD4+T-cells, DCs are secreting the cytokine, IL-16. According to the antigen, DCs are activating various cytokine genes. DCs' surface has receptors referred to as TLRs.

TLRs is identifying the pathogen's nature as well as sending signals for turning on specific cytokine genes. Also, the cytokine IL12 is inducing the T-cells for differentiating the sub-set Th1 cells. Also, the cytokine IL23 allow differentiating the T-cells into Th17 cells which are contracting with the extra cellular antigens. Besides, IL-4 is converting the T-cells into Th 2 Cells which are promoting productions of the antibodies via B-cells. Activated DCs are secreting the TGF-beta as well as IL-10 which is promoting the T-regulatory cells that are inducing immune responses.

DCs are initiating the innate immune response via NK T-cells, NKs and gamma-sigma T-cells. In addition, the stimulating viral improves the population of $\mathrm{pDC}$, and it is secreting the IFN-gamma as a response to the viral antigen challenges (Ahmad-Hasan et al., 2020). Besides, pDC cross key naïve CD8+T-cells through the transfer of the antigen into traditional DCs via exosomes. COVID-19 is interfering with secretory activities that are related to immune cells. Due to the acute infections, up regulations of chemokines results in heavy entry of monocytes, neutrophils and macrophages to sites that have been infected, leading to cytokine storm and tissue damage. With regard to acute cases, the cytokine storm is promoting pro-inflammatory cytokines synthesis IL1 beta, IL2, and IL6 promoting disease complications (Fu et al., 2020). INF production causes an increase in the acute situations (McKechnie, Blish, 2020).

\section{Effects of probiotic in COVID-19}

Probiotics are decreasing the infection's severity in upper respiratory tract and gastrointestinal tract through acting on adaptive and innate adaptive immune systems.

Now, using probiotic microorganisms as well as their metabolic products is representing a promising method to treat viral illnesses (Ryan et al., 2015). Also, the colonization related to intestinal epithelium via probiotic bacteria reduces the symptoms and rates of viral respiratory infections. This can be done via increasing the IgA expressing B-cells in the lymph nodes and colon along with an increase in the population of IL-23-expressing DCS and T-follicular helper cells (Kanauchi et al., 2018).

Gut and lung tissues are affected by COVID-19; therefore, inflammatory response is activated. It causes an increase in the proinflammatory cytokines IFN-gamma/TNF-alpha, which results in an increase in cytokine storm. The response might be because of the activation regarding Th1 cell responses in the tissues of the lungs (Letoranta et al., 2014). With regard to human gut environment, the dysbiosis in the microbiota of the gut leads to imbalances of Th1 and Th2, which is promoting the activation of pro inflammatory cytokine and lastly a cytokine storm in the lungs (Qian et al., 2017). After probiotics' management, there has been a colonization of "good bacteria" 
in the gut that results in shifting the balance between Th1/ Th2 cells and decreasing the cytokine storm as well as the disease's severity (Qian et al., 2017). Lately, it was identified that the medications with probiotic bacteria by means of Lactobacillus and Bifdobacteria are providing a considerable possibility of the recovery against COVID19 (Fanos et al., 2020).

With regard to various conditions of severe COVID-19, the occurrence of gastric symptoms has been reported. Due to the fact that the gastric symptoms are recorded, one might assume that SARS-CoV2 is interacting with the microbiota of the gut via gut/lung axis.

Today, it is indicated that it might be accountable for neuropsychiatric as well as cutaneous manifestations via the gut/skin, gut/brain and skin/brain axes (Salem et al., 2018; Charitos et al., 2020). SARS-CoV-2 results in local dysbiosis and, in try, the translocations that are related to microbial metabolites as well as toxins to other organs, like the gut. It must be indicated that the anti-virals and anti-biotics were majorly provided to patients experiencing SARS-CoV2, that might result in the dysbiosis of the gut microbiota. Thus, intestinal dysbiosis and dysregulated inflammation has been triggered not just via the infection. Patient's age or comorbidities might be behind the prime to higher disease severity and bad therapy results. Which could be an explanation for the increase in the COVID-19 severity because of the direct regulation regarding cross exchange between lung, intestine, skin and brain, which is increasing the immune dysregulation? (Angurana and Bansa, 2020; Gu et al., 2020; Riphagen et al., 2020).

Probiotic microbes are stimulating and modifying the immune system and reducing inflammations (Hardy et al., 2013). Bifidobacterium species and Lactobacillus species were the major traditional probiotics which might be utilized for balancing the expanded intestinal ecosystems in a fight of SARS-CoV2. In addition, the basis for utilizing probiotics and treating SARS-CoV2 is coming from a few experimental researches and indirect suggestions. Also, probiotics, including Lactobacillus can, achieve anti-viral actions, results in a eubiosis condition in the intestinal microbiota; therefore, contributing to the anti inflammatory effects and to super infection's prevention. It is recommended to use probiotics, along with their metabolites surfactant fatty acids (SCFAs) for supporting adaptive and innate immunity in patients who have SARS-CoV2, as adjuvant approach towards the complications. The basis for such line comes from various experimental indications (Ayawardena et al., 2020; Morais et al., 2020).

With regard to patients experiencing COVID-19, probiotics might allow restoring the changed gut microbiota, also contributes to healthy gut-lung axis. Also, they might be reducing the pathogens translocation via intestinal mucosa and avoid overlying infections. Probiotics might be interfering with the entry of the virus to host cells and with their replications. Bifidobacterium animals are inhibiting the replication of coronaviruses with anti-IL effects. Lactobacillus casei ATCC39392 is stimulating the expression of IL-17 throughout coronavirus gastroenteritis (Morais et al., 2020; Walton et al., 2020).

COVID-19 prompted intestinal dysbiosis has been enhanced via nutritional supports with the Lactobacillus acidophilus (Kagyama et al., 2020). Also, the SARS-CoV2 is damaging the epithelial layers of the gut. Lactobacillus plantarum is reinforcing viral damaged mucus epithelial barrier as well as enhancing the transportation of the probiotics to the lungs by the lung/gut axis and affecting the adaptive and innate immunity in the gut related lymphoid tissue, secondary lymphoid organs (Lundstrom, 2020; Zhang et al., 2020).

Lactobacillus plantarum shows anti-viral activity against the transmissible gastroenteritis virus, which is going to activate the anti-viral proteins through the signalling pathway of JAKSTAT, along with upregulating the interferon genes' expressions, leading to anti transmissible gastro-enteritis virus activities (Wang et al., 2019). Those reports are further indicating the efficiency of probiotic strains for treating the coronavirus.

Lactobacillus reuteri secretions such as reutericin, bacteriocins and reuteri-cyclin showing a rich anti-viral, anti-bacterial, anti-protozoan and anti-fungal activity. Lactobacillus reuteri producing tryptophan derived indole derivatives AhR and downregulating Thpok production to result in re-programming of CD 4+ IELs into DPIEL which is enhancing the anti-microbial peptides production (Reg3-lectins) for stimulating the innate immune responses against the intestinal pathogens that induce inflammations (Ang et al., 2016; Chen et al., 2018).

Lactobacillus salivarius in gut also improves the immunity towards the viruses inducing the inflammatory cytokines, IL10 (Zhai et al., 2020). Lactobacillus paracasei prevent SARS-COV-2 
binding with ACE-2 and its prevent entry the virus in the host cells and thereby resulted in the reduction of the infection chances (Rizo et al., 2020).

The ACE and di-peptidyl peptidase-4 that has been created by Lactobacillus lactis have immune-regulatory functions. Therefore, recombinant Lactobacillus vaccinations are planned towards the infection of COVID (Jiang et al., 2016). The Lactobacillus casei based oral vaccination towards transmissible gastro-enteritis corona virus stimulated Th-17 pathways. It resulted in the inhibition of the transmissible gastroenteritis corona virus. Numerous studies are performed all over the world, for the utilization of a variety of the Lactobacillus species as a new COVID-19 vaccination rector platform (Frederiksen et al., 2020; ISAPP, 2020).

Management of complex probiotic tablets Lactobacillus paracasei, Lactobacillus. acidophilus, Lactobacillus, Lactobacillus plantarum. delbrueckii, Bifidobacterium infantis, Bifidobacterium longum, Saccharomyces salivarius, and Bifidobacterium breve) to the children that have been admitted to ICU with the severe sepsis has been beneficial in increasing the antiinflammatory cytokines (TGF-beta-1, IL10) and decreasing the levels of the pro-inflammatory cytokines (IL-6, TNF-alpha, IL12 and IL-17) (Angurana, Bansal, 2020; Guan et al., 2020). Administrating over 20 probiotics established at improving the levels of the anti-inflammatory ILs and antiviral antibodies productions, resulting in the reduction of viral loads (Wang et al., 2013; Ballini et al., 2019). Coronaviruses maybe vulnerable as well to the probiotics. In the experimental models, they showed that the Enterococcus faecium NCIMB-10415 results in the increase of the nitric oxide, which results in the increase in the IL-6 and IL-8 expressions. Rather than that, Enterococcus faecium HDRf-1 has the ability for modifying the levels of the pro-inflammatory cytokines (TNF-alpha, IL1, IL-6, IL8, IL-12, IL-17) (Morais et al., 2020; Walton et al., 2020).

Amongst the elderly and immunocompromised patients, the group of the gut probiotics is typically low, which is why those patients endure severe COVID-19 impacts (Viana et al., 2020). The Probiotic anti-inflammatory secretions have the ability of regulating this inflammatory reaction through the co-supplementation of the personalized functional food that incorporates probiotic types (Antunes et al., 2020).

As therapeutic solutions are lingering for the COVID-19, care has been focused upon the sufficient nutritional therapies. Some of the latest reviews have highlighted the probiotics as adjunct alternatives amongst the therapies that are available for treatments for overcoming new corona virus (Aarti et al., 2020; Akour, 2020; Lisa et al., 2020).

In addition, Lactobacillus gasseri has an impact on the management of the purine as adjuvant nutritional treatment for helping the treatment of COVID-19 in weakening the viral replications (Morais et al., 2020). Numerous experimental researches have proven that probiotic Lactobacillus rhamnosus GG play a role in the secretion of protein $\mathrm{p} 40$, reducing the TNF, IL6, IFN-gamma, and chemo-attractant for the prevention of the inflammations in the epithelium of the gut (Yan et al., 2011).

A combination of Lactobacillus acidophilus, Lactobacillus reuteri, Lactobacillus casei as well as secretions of other probiotics had stimulated the DC functions and downregulated Th 1 , Th 2 and other factors, inducing the inflammation in lungs and gut via gut- lung axis link (Yang et al., 2020).

Plaza-Diaz et al. (2017) reported that the administrations of the probiotics has resulted in the reductions of systemic pro-inflammatory bio-markers in the non-gastrointestinal as well as the gastrointestinal conditions in the patients who have colitis after 6-8 weeks of therapy. The results of the RNA sequencing obtained by Feng et al. (2020) showed that the receptors of coronavirus, which include the angiotensin-converting enzyme 2 (ACE-2) for SARS-CoV and SARS-CoV2, have been highly expressed in the human enterocytes. It is interesting to notice that some of the potential target cells, which have constant expressions in small intestines, are constant at the same time as being changed continuously in the tissues of the lung. Which is why, the enterocytes can play the role of conserved cell reservoir for the corona viruses, a fact which has to draw attentions of the healthcare researchers to such SARS-CoV-2 infection site.

Lactobacilli have the ability block the viral particles' attachment to the human cells. Such distinctive characteristic had opened a platform for discovering the likelihood of the use of that microbe for the purpose of developing a local anti-viral nasal lactobacilli spray (Hanifi et al., 2020). Working on the nasal lactobacilli strains with the immune-stimulatory impacts for the purpose of using it as intra-nasal SARS-CoV2 vaccine adjuvants. Moreover, attempts for making the genetically engineered antigen-producing lactobacilli for the delivery of the vaccine are in progress 
(Leber, 2020). The viruses account for over $90 \%$ of the infections of the upper respiratory tract and numerous reports have informed potential of the probiotics of the lactobacilli on preventing the infections of the upper respiratory tract. SARS-CoV2 spread through the respiratory droplets attempts at developing anti-viral probiotic nasal spray can have a protective impact (Suet et al., 2020).

\section{Conclusion}

Probiotics enhance the immune system and modulate the immune response. Immunmudalotry benefit in SARS-COV-2 reduce the inflammation (tissue injury in respiratory tract) and decrease the symptoms of gastrointestinal. Probiotics stimulation of IgA in mucosal layer of gastrointestinal tract/respiratory tract and control the infection and this promoted an attention in probiotics of new generation to support immunity towards the treatment of COVID-19 viruses. Also, develop anti-viral probiotic (lactobacilli) nasal spray may be protective patient against infection.

\section{References}

Aarti et al., 2020 - Aarti, C., Martina, C., Khusro, A. (2020). Antimycobacterium, anticancer, and antiviral properties of probiotics: an overview. Microbes Inf. Dis, 1.

Ahmed-Hassan et al., 2020 - Ahmed-Hassan, H. et al. (2020). Innate immune responses to highly pathogenic coronaviruses and other significant respiratory viral infections. Front. Immunol. 11: 1979 .

Akour, 2020 - Akour, A. (2020). Probiotics and COVID-19: is there any link? Lett. Appl. Microbiol. 71(3): 229-234.

Ang et al., 2016 - Ang, L.Y. et al. (2016). Erratum to: antiviral activity of Lactobacillus reuteri Protectis against Coxsackievirus A and Enterovirus 71 infection in human skeletal muscle and colon cell lines. Virol. J. 13(1): 186.

Angurana, Bansal, 2020 - Angurana, S.K, Bansal, A. (2020). Probiotics and COVID-19: think about the link. Br. J. Nutr. $1 \mathrm{e} 26$.

Antunes et al., 2020 - Antunes, A.E.C., Vinderola, G., Xavier-Santos, D., Sivieri, K. (2020). Potential contribution of beneficial microbes to face the COVID-19 pandemic. Food Res. Int. 136.

Aziz et al., 2020 - Aziz, M., Perisetti, A., Lee-Smith, W.M., Gajendran, M., Bansal, P., Goyal, H. (2020). Taste Changes (Dysgeusia) in COVID-19: a systematic review and metaanalysis. Gastroenterology.

Ballin et al., 2019 - Ballini, A. et al. (2019). Effect of probiotics on the occurrence of nutrition absorption capacities in healthy children: a randomized double-blinded placebo-controlled pilot study. Eur. Rev. Med. Pharmacol. Sci. 23(19): 8645e57.

Charitos et al., 2020 - Charitos, I.A. et al. (2020). Special features of SARS-CoV-2 in daily practice. World J. Clin. 8(18): 3920e33.

Chen et al., 2018 - Chen, B. et al. (2018). Commensal BacteriaDependent CD8ab+ T cells in the intestinal epithelium produce antimicrobial peptides. Front. Immunol, 9: 1065.

Chen et al., 2020 - Chen, N. et al. (2020). Epidemiological and clinical characteristics of 99 cases of 2019 novel coronavirus pneumonia in Wuhan, China: a descriptive study. Lancet 395, 507-513.

D’Amico et al., 2020 - D’Amico, F., Baumgart, D.C., Danese, S., Peyrin-Biroulet, L. (2020). Diarrhea during COVID-19 infection: pathogenesis, epidemiology, prevention and management. Clin. Gastroenterol. Hepato.

De Almada et al., 2015 - De Almada, C.N., Nunes De Almada, C., Martinez, R.C., Sant'ana Ade, S. (2015). Characterization of the intestinal microbiota and its interaction with probiotics and health impacts. Appl Microbiol Biotechnol. 99: 4175-4199.

Dhar, Mohanty, 2020 - Dhar, D., Mohanty, A. (2020). Gut microbiota and Covid-19possible link and implications. Virus Res. 285: 198018.

Dockery et al., 2020 - Dockery, D.M., Rowe, S.G., Murphy, M.A., Krzystolik, M.G. (2020). The ocular manifestations and transmission of COVID-19: recommendations for prevention. J. Emerg. Med.

Drosten et al., 2003 - Drosten, C., Gunther, S. and Preiser, W. (2003). Identification of a novel coronavirus in patients with the severe acute respiratory syndrome. N. Engl. J. Med. 348: 1967-1976.

Fanos et al., 2020 - Fanos, V., Pintus, M.C., Pintus, R., Marcialis, M.A. (2020). Lung microbiota in the acute respiratory disease: from coronavirus to metabolomics. J. Pediatr. Neonatal. Individ. Med. 9: 90139. 
Feng et al., 2020 - Feng, Z., Wang, Y., Qi, W. (2020). The small intestine, an underestimated site of SARS-CoV-2 infection: from Red Queen effect to probiotics. Preprints.

Frederiksen et al., 2020 - Frederiksen, L.S.F., Zhang, Y., Foged, C., Thakur, A. (2020). The Long Road toward COVID-19 herd immunity: vaccine platform technologies and mass immunization strategies. Front. Immunol. 11: 1817.

Fu et al., $2020-F u, C$. et al. (2020). Plasmacytoid dendritic cells cross-prime naive CD8 T cells by transferring antigen to conventional dendritic cells through exosomes. Proc. Natl. Acad. Sci. U.S.A. 117(38): 23730-2374.

Gorbalenya et al., 2020 - Gorbalenya, A.E. et al. (2020). The species Severe acute respiratory syndrome-related coronavirus: classifying 2019-nCoV and naming it SARS-CoV-2. Nat. Microbiol. 5: 536-544.

$\mathrm{Gu}$ et al., $2020-G u, S$. et al. (2020). Alterations of the gut microbiota in patients with COVID-19 or H1N1 influenza. Clin Infect Dis. 4: ciaa709.

Guan et al., 2020 - Guan, W. et al. (2020). Clinical characteristics of coronavirus disease 2019 in China. N. Engl. J. Med. 382: 1708-1720.

Guimaraes et al., 2020 - Guimaraes, J.T. et al. (2020). Impact of probiotics and prebiotics on food texture. Curr Opin Food Sci. 33: 38-40.

Gupta et al., 2020 - Gupta, A. et al. (2020). Extrapulmonary manifestations of COVID-19. Nat. Med. 26(7): 1017-1032.

Hamid et al., 2020 - Hamid, S., Mir, M.Y., Rohela, G.K. (2020). Novel coronavirus disease (COVID-19): a pandemic (epidemiology, pathogenesis, and potential therapeutics). New Microbes New Infect. 35: 100679.

Hanifi et al., 2020 - Hanifi, G. et al. (2020). Lactobacilli species diversity in gut microbiota of renal failure patients. J. King Saud Univ. Sci. 32(4): 2365-2369.

Hardy et al., 2013 - Hardy, H. et al. (2013). Probiotics, prebiotics and immunomodulation of gut mucosal defences: homeostasis and immunopathology. Nutrients. 5: 1869-1912.

Hill et al., 2014 - Hill, C. et al. (2014). Expert consensus document. The International Scientific Association for Probiotics and Prebiotics consensus statement on the scope and appropriate use of the term probiotic. Nat Rev Gastroenterol Hepatol. 11: 506-514.

Hoffmann et al., 2020 - Hoffmann, M. et al. (2020). SARS-CoV-2 Cell Entry Depends on ACE2 and TMPRSS2 and Is Blocked by a Clinically Proven Protease Inhibitor. Cell. 181: 271-28.

Hopkins et al., 2020 - Hopkins, C., Surda, P., Whitehead, E., Kumar, B.N. (2020). Early recovery following new onset anosmia during the COVID-19 pandemic - an observational cohort study. J. Otolaryngol. Head Neck Surg. 49: 26.

Huang et al., 2020 - Huang, C. et al. (2020). Clinical features of patients infected with 2019 novel coronavirus in Wuhan, China. Lancet. 395: 497-506.

Hulswit et al., 2016 - Hulswit, R.J., de Haan, C.A., Bosch, B.J. (2016). Coronavirus spike protein and tropism changes. Adv Virus Res. 96: 29-57.

ISAPPBD, 2020 - International Scientific Association of Probiotics and Prebiotics Board of Directors. (2020). How some probiotic scientists are working to address COVID-19 [Online]. ISAPP.

Jiang et al., 2020 - Jiang, F. et al. (2020). Review of the clinical characteristics of coronavirus disease 2019 (COVID-19). J. Gen. Intern. Med. 4: 1-5.

Kageyama et al., 2020 - Kageyama, Y., Akiyama, T., Nakamura, T. (2020). Intestinal dysbiosis and probiotics in COVID-19. J. Clin. Trials. 10: 421.

Kanauchi et al., 2018 - Kanauchi, O., Andoh, A., AbuBakar, S., Yamamoto, N. (2018). Probiotics and Para probiotics in viral infection: clinical application and effects on the innate and acquired immune systems. Curr. Pharm. Des. 24: 710.

Khaneghaha et al., 2020 - Khaneghaha, A.M. et al. (2020). Interactions between probiotics and pathogenic microorganisms in hosts and foods: a review. Trends Food Sci Technol. 95: 205-218.

Kiousi et al., 2019 - Kiousi, D.E. et al. (2019). Probiotics in extraintestinal diseases: current trends and new directions. Nutrients. 11: 788.

Kopel et al., 2020 - Kopel, J. et al. (2020). Clinical Insights into the gastrointestinal manifestations of COVID-19. Dig Dis Sci. 65: 1932-1939.

Kuri-Cervantes et al., 2020 - Kuri-Cervantes, L., Pampena, M.B., Meng, W., Rosenfeld,A.M., Ittner, C.A.G., Weisman, A.R., Agyekum, R.S., Mathew, D., Baxter, A.E., 
Vella, L.A. et al. (2020). Comprehensive mapping of immune perturbations associated with severe COVID-19. Sci. Immunol.

Lamers et al., 2020 - Lamers, M.M. et al. (2020). SARS-CoV-2 productively infects human gut enterocytes. Science.

Lao et al., 2020 - Lao, W.P., Imam, S.A., Nguyen, S.A. (2020). Anosmia, hyposmia, and dysgeusia as indicators for positive SARS-CoV-2 infection. World J. Otorhinolaryngol-Head Neck Surg.

Lazarian et al., 2020 - Lazarian, G. et al. (2020). Autoimmune haemolytic anaemia associated with COVID-19 infection. Br. J. Haematol.

Lebeer, 2020 - Lebeer, $S$. (2020). University of Antwerp, Belgium: Relevance of the airway microbiome profile to COVID-19 respiratory infection and using certain lactobacilli to enhance delivery or efficacy of vaccines. ISAPP Science Blog, News.

Lehtoranta et al., 2014 - Lehtoranta, L., Pitkäranta, A., Korpela, R. (2014). Probiotics in respiratory virus infections. Eur. J. Clin. Microbiol. Infect. Dis. 33: 1289-1302.

Li et al., 2005 - Li, F., Li, W., Farzan, M., Harrison, S.C. (2005). Structural biology: structure of SARS coronavirus spike receptor-binding domain complexed with receptor. Science. (80- ), 309: 1864-1868.

Li, $2012-L i, F$. (2012). Evidence for a common evolutionary origin of coronavirus spike protein receptor-binding subunits. J. Virol. 86: 2856-2858.

Liisa et al., 2020 - Liisa, L., Sinikka, L., Markus, J.L. (2020). Role of probiotics in stimulating the immune system in viral respiratory tract infections: a narrative review. Nutrients. 12: 3163 .

Lu et al., 2015 - Lu, G., Wang, Q., Gao, G.F. (2015). Bat-to-human: spike features determining "host jump" of coronaviruses SARS-CoV, MERS-CoV, and beyond. Trends Microbiol. 23: 468-478.

Lundstrom, 2020 - Lundstrom, K. (2020). Coronavirus pandemic-therapy and vaccines. Biomedicines. 8(5): 109.

McKechnie, Blish, 2020 - McKechnie, J.L., Blish, C.A. (2020). The innate immune system: fighting on the front lines or fanning the flames of COVID-19? Cell Host Microbe. 27(6): 863-869.

Mermelstein, 2020 - Mermelstein, S. (2020). Acute anosmia from COVID-19 infection. Pract. Neurol.

Morais et al., 2020 - Morais, A.H.A., Passos, T.S., Maciel, B.L.L., da Silva-Maia, J.K. (2020). Can probiotics and diet promote beneficial immune modulation and purine control in coronavirus infection? Nutrients. 12(6): 1737.

Neri-Numa et al., 2020 - Neri-Numa, A.I. et al. (2020). Natural prebiotic carbohydrates, carotenoids and flavonoids as ingredients in food systems. Curr Opin Food Sci. 33: 98-107.

Nunan et al., 2020 - Nunan, D. (2020). Loss of Smell and Taste As Symptoms of COVID-19: What Does the Evidence Say? CEBM, Oxford.

Plaza-Diaz et al., 2017 - Plaza-Diaz, J., Ruiz-Ojeda, F.J., Vilchez-Padial, L.M., Gil, A. (2017). Evidence of the anti-inflammatory effects of probiotics and synbiotics in intestinal chronic diseases. Nutrients. 9: 555.

Qi et al., 2020 - Qi, F., Qian, S., Zhang, S., Zhang, Z. (2020). Single cell RNA sequencing of 13 human tissues identify cell types and receptors of human coronaviruses. Biochem. Biophys. Res. Commun. 526: 135-140.

Qian et al., 2017 - Qian, W., et al. (2017). The C-terminal efector domain of non-structural protein 1 of influenza A virus blocks IFN- $\beta$ production by targeting TNF receptor-associated factor 3. Front. Immunol. 8: 779-779.

Reguera et al., 2012 - Reguera, J. et al. (2012). Structural bases of coronavirus attachment to host aminopeptidase $\mathrm{N}$ and its inhibition by neutralizing antibodies. PLoS Pathog. 8: e1002859.

Riphagen et al., 2020 - Riphagen, S. et al. (2020). Hyper inflammatory shock in children during COVID-19 pandemic. Lancet. 95: $1607 \mathrm{e} 8$.

Rizzo et al., 2020 - Rizzo, P. et al. (2020). COVID-19 in the heart and the lungs: could we "Notch" the infammatory storm? Basic Res Cardiol. 115(3): 31.

Roobab et al., 2020 - Roobab, U. et al. (2020). Sources, formulations, advanced delivery and health benefits of probiotics. Curr Opin Food Sci. 32: 17-28.

Ryan et al., 2015 - Ryan, P.M. et al. (2015). Sugarcoated: exopolysaccharide producing lactic acid bacteria for food and human health applications. Food Funct. 6: 679-693. 
Salem et al., 2018 - Salem, I., Ramser, A., Isham, N., Ghannoum, M.A. (2018). The gut microbiome as a major regulator of the gut-skin Axis. Front. Microbiol. 10(9): 1459.

Santacroce, 2020 - Santacroce, L. (2020). Letter in response to the article "Enhancing immunity in viral infections, with special emphasis on COVID-19: a review" (Jayawardena et al.). Diabetes Metab Syndr. 14(5): 927.

Su et al., $2020-S u, M$. et al. (2020). Probiotics for the prevention of ventilator associated pneumonia: a meta-analysis of randomized controlled trials. Respir. Care. $65: 5$.

Viana et al., 2020 - Viana, S.D., Nunes, S., Reis, F. (2020). ACE2 imbalance as a key player for the poor outcomes in COVID-19 patients with age-related comorbidities role of gut microbiota dysbiosis. Ageing Res. Rev. 62.

Walton et al., 2020 - Walton, G.E., Gibson, G.R., Hunter, K.A. (2020). Mechanisms linking the human gut microbiome to prophylactic and treatment strategies for COVID-19. Br. J. Nutr. 9: 1e36.

Wang et al., 2013 - Wang, G. et al. (2013). Effect of enteral nutrition and ecoimmunonutrition on bacterial translocation and cytokine production in patients with severe acute pancreatitis. J.Surg. Res. 183(2): 592e7.

Wang et al., 2019 - Wang, K. et al. (2019). Anti-TGEV miller strain infection efect of Lactobacillus plantarum supernatant based on the jAKSTAT1 signaling pathway. Front Microbiol. 10: 2540.

Wang et al., 2020 - Wang, D. et al. (2020). Clinical Characteristics of 138 Hospitalized Patients with 2019 Novel Coronavirus-Infected Pneumonia in Wuhan, China. JAMA - J Am Med Assoc. 323: 1061-1069.

Wilk et al., 2020 - Wilk, A.J. et al. (2020). A single-cell atlas of the peripheral immune response in patients with severe COVID-19. Nat Med. 26: 1070-1076.

Wu et al., $2009-W u, K ., L i, W$. ., Peng, G. and Li, F. (2009). Crystal structure of NL63 respiratory coronavirus receptor-binding domain complexed with its human receptor. Proc Natl Acad Sci USA. 106: 19970-19974.

Wu et al., $2020-W u$, F. et al. (2020). A new coronavirus associated with human respiratory disease in China. Nature.

Xiong et al., 2020 - Xiong, Y. et al. (2020). Transcriptomic characteristics of bronchoalveolar lavage fluid and peripheral blood mononuclear cells in COVID-19 patients. Emerging Microbes Infect. 9(1): 761-770.

$\mathrm{Xu}$ et al., $2020-X u, Z$. et al. (2020). Pathological findings of COVID-19 associated with acute respiratory distress syndrome. Lancet Respir. Med. 8: 420-422.

Yan et al., 2011 - Yan, F. et al. (2011). Colon-specific delivery of a probiotic-derived soluble protein ameliorates intestinal inflammation in mice through an EGFR dependent mechanism. J. Clin. Invest. 21(6): 2242-2253.

Yang et al., 2020 - Yang, D. et al. (2020). Attenuated interferon and proinflammatory response in SARS-CoV-2-infected human dendritic cells is associated with viral antagonism of STAT1 phosphorylation. J. Infect. Dis. 222(5):734-745.

Zaki et al., 2012 - Zaki, A.M. et al. (2012). Isolation of a novel coronavirus from a man with pneumonia in Saudi Arabia. N. Engl. J. Med. 367: 1814-1820.

Zendeboodi et al., 2020 - Zendeboodi, F., Khorshidian, N., Mortazavian, A.M., Da Cruz, A.G. (2020). Probiotic: conceptualization from a new approach. Curr Opin Food Sci. 32: 103-123.

Zhai et al., 2020 - Zhai, O. et al. (2020). Screening of Lactobacillus salivarius strains from the feces of Chinese populations and the evaluation of their effects against intestinal inflammation in mice. Food Funct. 11(1): 221-235.

Zhang et al., 2020 - Zhang, N., Li, C., Niu, Z., Kang, H., Wang, M. (2020). Colonization and immunoregulation of Lactobacillus plantarum BF_15, a novel probiotic strainfrom the faces of breast-fed infants. Food Funct. 11: 3156-3166.

Zhou et al., 2020 - Zhou, . et al. (2020). Acute SARS-CoV-2 infection impairs dendritic cell and $T$ cell responses. Immunity.

Zhu et al., $2020-Z h u, N$. et al. (2020). A novel coronavirus from patients with pneumonia in China, 2019. N. Engl. J. Med. 382: 727-733.

Zhuang et al., 2019 - Zhuang, H. et al. (2019). Dysbiosis of the gut microbiome in lung cancer. Front Cell Infect Microbiol. 